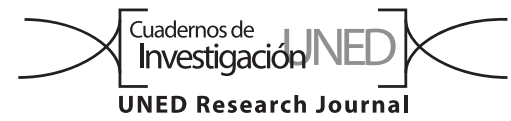

\title{
Género y cambio climático: retrospectiva y retos
}

\section{Lorena Aguilar Revelo}

1. Unión Internacional para la Conservación de la Naturaleza (UICN), Washington D.C., Estados Unidos de América; equidad2000@yahoo.com

Recibido 28-VII-2018 • Corregido 24-IX-2018 • Aceptado 14-I-2019

\begin{abstract}
Gender and climate change: retrospective and challenges. Climate change is a problem of all human kind; however, there is gender inequality in the impacts of climate change, with greater impacts on women. In 2010, the International Union for Conservation of Nature (IUCN) established a methodology for the development of Gender and Climate Change Action Plans (CCGaps) with the aim to promote a comprehensive and multisectoral approach towards climate change. The CCGaps process includes: 1) Information inventory; 2) Leveling the field; 3) Taking all voices into account; 4) Prioritizing; and 5) Implementation/action. To date, this methodology has been used by 20 national governments and in three regional processes. The methodology established by the IUCN to develop the CCGaps represents an innovative way to mainstream gender perspective. It is based on strengthening the capacity of the interest groups to build adequate, non-conventional, concrete, practical and innovative solutions. A CCGaps focuses on key sectors identified by each country and recognizes gender equality as a transformative engine at a national level.
\end{abstract}

Key words: IUCN, gender-responsive climate policy, adaptation.
RESUMEN: El cambio climático es un problema de toda la humanidad; sin embargo, hay inequidad de género en los impactos del cambio climático, donde las mujeres son más impactadas. En el 2010, la Unión Internacional para la Conservación de la Naturaleza (UICN) desarrolló una metodología para elaborar Planes de Acción de Género y Cambio Climático (PAGcc) con el objetivo de promover un enfoque integral y multisectorial ante el cambio climático. El proceso de un PAGcc incluye: 1) Inventario de información; 2) Nivelar el terreno; 3) Tomar en cuenta la diversidad de voces; 4) Priorizar; y 5) Implementación/acción. A la fecha, esta metodología ha sido utilizada por 20 gobiernos nacionales y en tres procesos regionales. La metodología desarrollada por la UICN para elaborar los PAGcc presenta una forma innovadora para transversalizar la perspectiva de género. Se basa en el fortalecimiento de capacidades de los grupos de interés para construir soluciones apropiadas, no convencionales, concretas, prácticas e innovadoras. Un PAGcc se centra en sectores claves identificados por cada país y reconoce la igualdad de género como un motor transformador a nivel nacional.

Palabras clave: UICN, política climática género-responsiva, adaptación.
El análisis histórico en este artículo, no deja duda alguna sobre los avances significativos de los últimos veinte años en la forma en que la política internacional está abordando la complejidad y la interseccionalidad de los retos globales para el desarrollo sostenible. Sin embargo, mientras que la política global puede reflejar mejor la complejidad del mundo, la eficacia de los procesos multilaterales que conduzcan a la implementación de acciones exige estrategias claras y nuevas medidas para crear un cambio real.

Ante esta necesidad, este capítulo hace un recuento histórico con relación a la metodología desarrollada por la Unión Internacional para la Conservación de la Naturaleza (UICN) para elaborar Planes de Acción en Género y Cambio Climático (PAGcc). Los PAGcc son una forma innovadora para transversalizar la perspectiva de género. Se basa en el fortalecimiento de capacidades de los grupos de interés para construir soluciones apropiadas, no convencionales, concretas, prácticas e innovadoras. Los PAGcc fomentan un enfoque integral, que abarca desde la garantía de la igualdad de género en el marco de políticas sectoriales hasta la reducción de barreras en prácticas institucionales a actividades que son impulsadas por mujeres líderes y empresarias comprometidas en respuestas al cambio climático para generar comunidades y países más resilientes. Sin embargo, los retos hacia futuro son muchos y de diversa índole de ahí la necesidad de concluir este documento con una serie de recomendaciones.

\section{Retrospectiva}

La Convención Marco de las Naciones Unidas sobre Cambio Climático (CMNUCC) se creó en 1992 para abordar el tema de cambio climático con el objetivo 
de estabilizar las concentraciones de gases de efecto invernadero (GEI).

Originalmente la CMNUCC fue un espacio tecnocrático que proponía políticas de forma vertical centrada principalmente en la mitigación y se caracterizó, en su inicio, por adolecer de referencias sobre temas sociales. Sin embargo, a partir del 2009, las Partes ante la CMNUCC dan un cambio importante al reconocer las consideraciones sociales y de género en decisiones y posiciones. Los factores que han propiciado este movimiento son múltiples e interrelacionados. Algunos de estos factores están vinculados con la evolución de los derechos humanos y el desarrollo sostenible, otros con la incidencia de la sociedad civil, gobiernos, organizaciones internacionales y de investigación para asegurar que la política climática sea capaz de abordar los desafíos de un desarrollo justo y equitativo, junto con su mandato de incidir en la mitigación y adaptación al cambio climático.

La evolución de esta política también se ha visto influenciada por factores tales como los impactos cada vez más evidentes en todo el mundo, del cambio climático en las comunidades locales; el vínculo entre las crisis ambientales y económicas de los últimos años; y el creciente reconocimiento de que la igualdad de género es parte integral del desarrollo y del bienestar de todas las personas, de todas las edades y grupos étnicos.

Otro aspecto crítico ha sido la participación directa de las mujeres y su liderazgo en la política a nivel internacional, así como dentro de la CMNUCC. La participación de las mujeres en las delegaciones nacionales en las sesiones de la COP y otras reuniones de la CMNUCC ha variado desde un $29 \%$ de mujeres que asistieron a la COP 186 en 2012 hasta un $42 \%$ asistiendo a las sesiones 44a y 46a de los órganos subsidiarios en 20167 y 2017,8 respectivamente. La mayor participación de mujeres en una COP se registró después de la adopción de la decisión 23 / CP.18; en la COP 19 y COP 20, el porcentaje de mujeres que asistieron fue del $36 \%$, frente al $29 \%$ en COP 18,9 . Sin embargo, en la COP 21 y la COP 22, el porcentaje de mujeres cayó del 36\% al 32\%. El porcentaje más alto registrado de mujeres jefes de delegación fue en la CP 22 con el 27\% (CMNUCC, 2017).

\section{La GGCA en acción: Uniendo fuerzas para facilitar el cambio}

En 2007 en Bali, en el marco de la Conferencia de las Partes (COP) 13, cuatro organizaciones con larga trayectoria de incidencia en cuanto a género y medio ambiente definieron una estrategia común para avanzar la integración de los aspectos de igualdad de género y empoderamiento de las mujeres en los diversos espacios sobre el cambio climático. La Unión Internacional para la Conservación de la Naturaleza (UICN), el Programa de las Naciones Unidas para el Desarrollo ( PNUD), el Programa de las Naciones Unidas para el Medio Ambiente (PNUMA) y la organización Women's Environment and Development Organization (WEDO), instauraron oficialmente la Alianza Global de Género y Cambio Climático (GGCA, por sus siglas en inglés) para asegurar que la toma de decisiones políticas y programas de cambio climático en todos los niveles sean género responsivas. La GGCA es un espacio único que convoca a organizaciones intergubernamentales, no gubernamentales y agencias de las Naciones Unidas. Al presente, la GGCA cuenta con alrededor de 100 miembros de todas las regiones del mundo.

La GGCA es reconocida como la Alianza más influyente en los espacios de la CMNUCC. Gracias al soporte técnico brindado a las Partes, estos han aprobado más de 50 decisiones con relación a género y a los derechos humanos.

Desde 2008 la GGCA ha contado con el apoyo financiero del Gobierno de Finlandia y otros aliados que han financiado proyectos específicos a través del tiempo.

\section{Género en la CMNUCC: Desde la "invisibilidad de género" hasta una política género responsiva}

A finales de 2015, la CMNUCC contaba con más de 45 decisiones en materia de género. Del total de las mismas (excluyendo el nuevo texto del acuerdo marco)
8 explícitamente se refieren solamente al balance de género y a incrementar la participación de las mujeres en los consejos y órganos; 21 decisiones proponen un enfoque género sensible; una decisión ve a las mujeres como grupo vulnerable; 13 decisiones reconocen tanto la necesidad de un balance y un enfoque de género; y 2 
decisiones adoptan un enfoque género sensible y visión de las mujeres como grupo vulnerable.

La figura 1 muestran información sobre las decisiones y los temas referentes a género incluidos desde la COP 1 hasta la COP 21.

Adicionalmente, el Acuerdo de París (UNFCCC, 2016) cuenta con tres referencias. Ello constituye un avance sin precedente, pues el primer Acuerdo Marco no tenía ninguna referencia al tema de género ni de derechos humanos. Los textos en referencia se encuentran abajo:
Preámbulo. Reconociendo que el cambio climático es un problema de toda la humanidad y que, al adoptar medidas para hacerle frente, las Partes deberían respetar, promover y tener en cuenta sus respectivas obligaciones relativas a los derechos humanos, el derecho a la salud, los derechos de los pueblos indígenas, las comunidades locales, los migrantes, los niños, las personas con discapacidad y las personas en situaciones vulnerables y el derecho al desarrollo, así como la igualdad de género, el empoderamiento de la mujer y la equidad intergeneracional . (UNFCCC, 2016, p. 1)

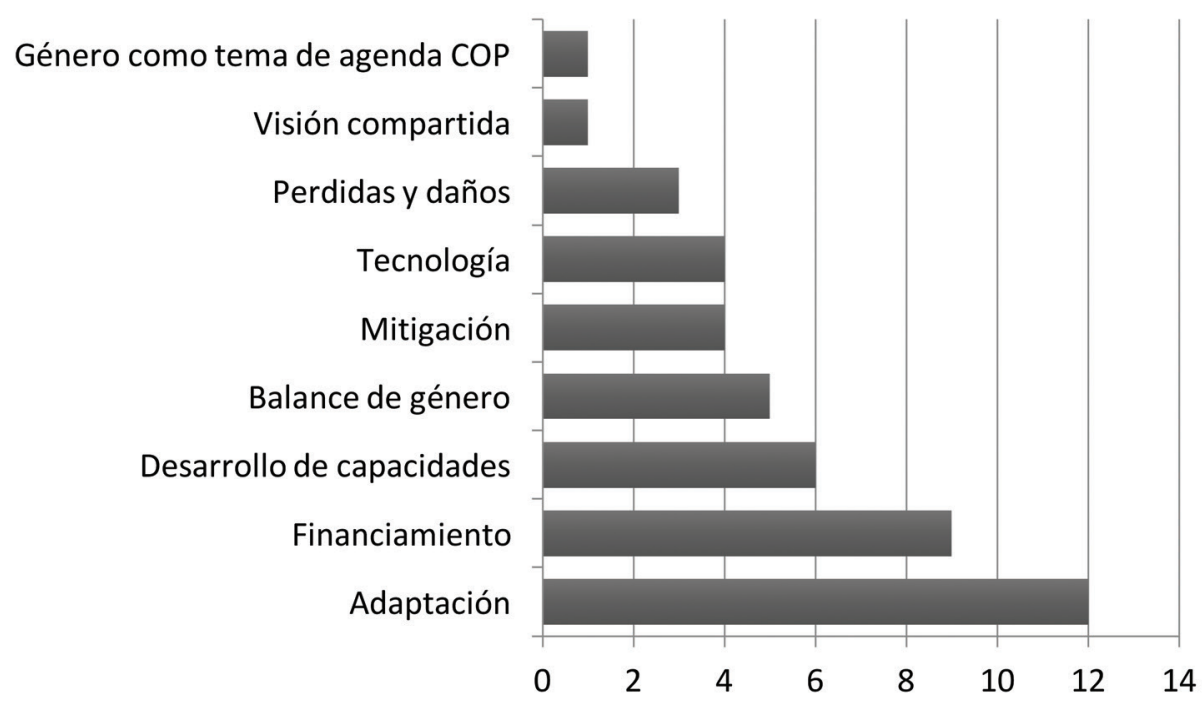

Fig. 1. Número de decisiones que abordan género por tema (COP1 - COP21).

Adaptación. Artículo 7, apartado 5. 5. Las Partes reconocen que la labor de adaptación debería llevarse a cabo mediante un enfoque que deje el control en manos de los países, responda a las cuestiones de género y sea participativo y del todo transparente, tomando en consideración a los grupos, comunidades y ecosistemas vulnerables.... (UNFCCC, 2016, p. 7).

Artículo 11, párrafo 2. El fomento de la capacidad debería estar bajo el control de los países, basarse en las necesidades nacionales y responder a ellas, y fomentar la implicación de las Partes, en particular de las que son países en desarrollo, incluyendo en los planos nacional, subnacional y local. El fomento de la capacidad debería guiarse por las lecciones aprendidas, también en las actividades en esta esfera realizadas en el marco de la Convención, y debería ser un proceso eficaz e iterativo, que sea participativo y transversal y que responda a las cuestiones de género (UNFCCC, 2016, p.11).

El cuadro 1 muestra cómo las decisiones bajo la CMNUCC han progresado en los últimos años. 


\section{CUADRO 1}

Línea de tiempo: transversalización de género en la CMNUCC

Año

1995, Berlín CMNUCC Igualdad de Género y Derechos de las Mujeres

La primera COP de la CMNUCC definió el 1a Conferencia de mujeres dentro de en la CMNUCC.

Protocolo de Kioto (PK), estableció el proceso de negociación para el fortalecimiento de los compromisos de los países desarrollados.

1997, Kioto COP3 Adopción histórica del PK: Es el primer Primera "Asamblea de Mujeres". tratado mundial para la reducción de gases de efecto invernadero.

2001, Marrakech

COP7 Acuerdo de Marrakech establece el Primeras decisiones y referencias de género: Decisión 36 / CP.7 sobre escenario para la ratificación del Protocolo participación de las mujeres en la representación de las Partes en los órganos de Kioto. establecidos en virtud de la CMNUCC o el Protocolo de Kioto, y la Decisión 28 / CP.7 sobre las Directrices para la preparación de la de las Planes Nacionales para Adaptación (PNA) estableciendo que los mismos deben considerar la igualdad de género.

\section{PUNTO DE QUIEBRE}

2008, Poznan

2009, Copenhague

COP15 falla en producir un resultado ambicioso en línea con el Plan de Acción de Bali, en su lugar se tiene el Acuerdo de Copenhague.
COP14 toma medidas para ayudar a los UICN como parte de la GGCA publicó el Manual de Capacitación en países en desarrollo, por ejemplo, el Fondo Género y Cambio Climática (Aguilar, 2009) y organizó la primera sesión de de Adaptación bajo el Protocolo de Kioto y orientación sobre las dimensiones de género en el cambio climático para el Programa Estratégico de Poznan sobre transferencia de tecnologías.

las delegaciones de los países, lo que ayudó a incrementar la conciencia y el entendimiento sobre el tema entre las Partes.
La GGCA brinda apoyo técnico a delegados para incidir para la inclusión de género en el texto.

La GGCA continúa dando orientación a los delegados de las Partes sobre el vínculo entre género y cambio climático.

En el período previo a Copenhague, activistas de género presionaron con éxito para la inclusión del lenguaje de género en todas las esferas de las negociaciones; las Partes incluyeron el término de género en el texto de los documentos de negociación.

Se le da estatus provisional al grupo de interés de las "Mujeres."

2010, Cancún

Resultados de la COP16: Acuerdo de Cancún, con un paquete integral para ayudar a las naciones en desarrollo a enfrentar el cambio climático. El Fondo Verde para el Clima (FV), el Mecanismo de Tecnología y el Marco de Los Acuerdos de Cancún incluyen 8 referencias a las mujeres y género en las Adaptación de Cancún se crean. siete secciones en los textos de decisión. Se logran referencias específicas bajo adaptación, REDD+, Transferencia de Tecnología y Desarrollo de Capacidades.

2011, Durban

En la COP17, las Partes se comprometen a finalizar un nuevo acuerdo universal sobre cambio climático en el 2015.
Las referencias a género en el FV y el Centro de Tecnología para el Clima y la Red muestran evolución en la comprensión de consideraciones sociales y de género.

Se le otorga al grupo de interés de las "Mujeres" estatus formal como un grupo de Circunscripción ante la CMNUCC. 
2012, Doha

2013, Varsovia

2015, Ginebra y Paris

2014, Lima
En la COP18, las Partes acuerdan trabajar hacia un acuerdo universal sobre cambio climático al 2015 y encontrar maneras para ampliar los esfuerzos antes del 2020 y más allá de los compromisos existentes para reducir las emisiones.

La Enmienda Doha lanza un segundo período de compromiso para el Protocolo de Kioto.

La Secretaría de la CMNUCC lanza la iniciativa "Momentum for Change", incluyendo un eje específico para mujeres.

COP19 produce los resultados de Varsovia, incluyendo un libro de reglas para REDD $+y$ un mecanismo sobre pérdidas y daños.
COP18 vio un aumento en la atención a los aspectos de género y cambio

climático: Se oficializa el "Día de Género" organizado por la Secretaría de la Convención.

Se logran decisiones de género bajo: PAN, pérdidas y daños, Centro de Tecnología del Clima, criterios para la junta asesora del mecanismo de la tecnología, y el nuevo programa de trabajo sobre el Artículo 6 en educación y la información.

Decisión 23 / CP.18: promover el balance de género y mejorar la participación de las mujeres en las negociaciones de la CMNUCC y hacer de género un tema permanente del programa de la COP.
Las decisiones incluyen lenguaje sobre la igualdad de género y las consideraciones sociales en catorce áreas de programas, entre ellos: transferencia de tecnología, finanzas y adaptación y la importancia de la información desagregada por sexo en el nuevo mecanismo establecido para pérdida y daños.

COP19 fue sede del primer taller sobre género y cambio climático (UNFCCC, 2015).

En la COP20, las Partes producen una decisión sobre la presentación de Contribuciones Prevista y Determinada a Nivel Nacional (INDCs por sus siglas en inglés) y presentan el texto base para el nuevo acuerdo.

Texto adjunto del nuevo acuerdo climático incluye referencias a género en la exposición de motivos, en las secciones sobre adaptación, finanzas y el desarrollo de capacidades, y también a través de siete decisiones en virtud de los Órganos Subsidiarios.

Las Partes adoptaron la Decisión 18 / CP.20, la cual es la decisión más importante de los últimos años pues sienta las bases para implementar las decisiones acordadas en la última década.

México - apoyado por Uganda y Chile- inicia las sesiones de negociación con la manifestación: "Las Partes deben respetar los derechos humanos y la igualdad de género en la aplicación de todas las políticas y acciones climáticas." acuerdo 2015; las negociaciones concluyen con la adopción del " texto de Ginebra" que sirvió de base para las discusiones la elaboración de Acuerdo Paris.
La Unión Europea, los países menos desarrollados y el Grupo Africano presentaron propuestas en materia de igualdad de género.

A mediados de año, el lenguaje de la igualdad de género, derechos de las mujeres, la participación pública y los pueblos indígenas se integra en todas las áreas del texto de negociación.

En la Reunión de la Junta Directiva del FV la política de género y su Plan de Acción se adopta haciendo a este mecanismo financiero el primero en transversalizar género antes de que el fondo entre en vigor.

La COP 21 aprueba el nuevo Acuerdo Marco, que incluye tres referencias al tema de género y los derechos humanos

Fuente: Blomstrom y Burns (2015, p. 67-71). 
No hay duda de que se han logrado avances significativos en los últimos veinte años en la forma en que la política internacional está abordando la complejidad y la interseccionalidad de los retos globales para el desarrollo sostenible.

Sin embargo, mientras que la política global puede reflejar mejor la complejidad del mundo, la eficacia de los procesos multilaterales que conduzcan a la implementación de acciones exige estrategias claras y nuevas medidas para crear un cambio real.

Ello hace necesario una clara definición tanto práctica como metodológica para que la política climática sea género-responsiva'.

En primer lugar, se deben convertir las "buenas intenciones de los acuerdos o políticas" en acciones a nivel regional, nacional y local. En segundo lugar, es necesario identificar, en los ámbitos alternativos de influencia, los espacios necesarios para crear conciencia y capacidad para abordar los vínculos entre género y cambio climático.

Algunos países han venido avanzando al integrar enfoques participativos para garantizar que la diversidad de necesidades, experiencias, conocimientos y capacidades, se incluyan en las políticas y procesos de planificación relacionados con el cambio climático a nivel nacional. Ejemplo de ello es el desarrollo de los Planes de Acción de Género y Cambio Climático (PAGcc) que se detalla a continuación.

\section{Planes de Acción de Género y Cambio Climático (PAGcc)}

En el 2010, la UICN desarrolló una metodología para elaborar PAGcc con el objetivo de promover un enfoque integral y multisectorial ante el cambio climático. A la fecha, esta metodología ha sido utilizada por 20 gobiernos nacionales y tres procesos regionales. El Cuadro 2 incluye los planes de acción formulados, el año en que fueron creados, así como los temas que se abordaron.

Siguiendo la misma metodología de los PAGcc, también se han elaborado hojas de ruta de "Género y REDD+" en cuatro países: Ghana, Uganda, Cameron y
México (http://genderandenvironment.org/resource/ gender-and-redd-roadmaps/).

\section{METODOLOGÍA}

La metodología desarrollada por la UICN para elaborar los PAGcc presenta una forma innovadora para transversalizar la perspectiva de género. Se basa en el fortalecimiento de capacidades de los grupos de interés para construir soluciones apropiadas, no convencionales, concretas, prácticas e innovadoras. Los PAGcc fomentan un enfoque integral, que abarca desde la igualdad en el proceso de formulación de políticas de los sectores técnicos, hasta la reducción de barreras en prácticas institucionales a actividades que son impulsadas por mujeres líderes y empresarias comprometidas en respuestas al cambio climático para generar comunidades y países más resilientes. Un PAGcc va más allá de ver a las mujeres como víctimas o grupo vulnerable. Más bien reconoce la igualdad de género como un motor transformador a nivel nacional.

Un PAGcc es el resultado de una serie de pasos: investigación documental, entrevistas con los responsables de crear políticas, consultas con los interesados y las revisiones por grupos de pares, entre otros. Un PAGcc se centra en sectores claves identificados por cada país, incluyendo, pero no limitado a: agua, agricultura, salud, mitigación (energía y bosques), adaptación, reducción del riesgo a desastres (RRD), infraestructura, manejo de residuos, turismo y manejo costero entre otros. De esta manera, el proceso se lleva a cabo con la participación de diversos sectores y actores interesados. La experiencia ha demostrado que a menudo representa la primera vez que personal técnico de los diferentes sectores, como el de agua, energía o las instancias responsables de la política de igualdad de género, tienen la oportunidad de dialogar y desarrollar capacidades y formular propuestas en aspectos clave relacionados con género y cambio climático.

La participación de organizaciones y redes de mujeres -independientemente de su nivel de conocimiento acerca del cambio climático- también es clave, ya que contribuyen a la formulación de políticas con base en la

1. Género-responsivo(a): Identificar, comprender e implementar acciones para cerrar las brechas de género y superar los sesgos de género históricos en las políticas e intervenciones. El ser género-responsivo en su aplicación, significa contribuir de manera pro-activa e intencional a promover la igualdad de género. Una política, programa, plan o proyecto género-responsivo se propone no sólo'no ocasionar daño' sino 'aspirar a algo mejor'. 


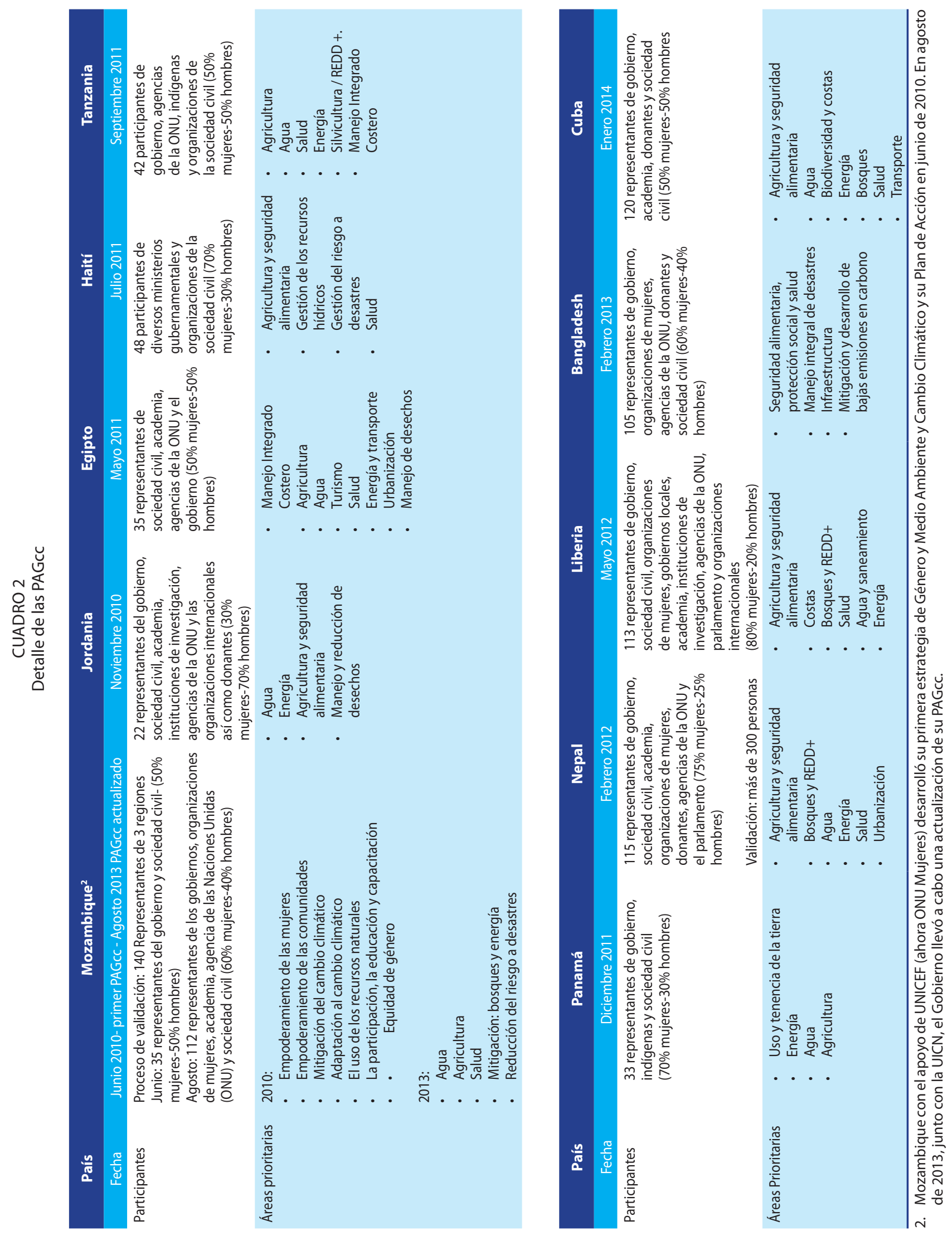

(c) open 2 Access Cuadernos de Investigación UNED (ISSN digital: 1659-441X) Vol. 11 (1) Núm. especial: S89-S102, marzo 2019 


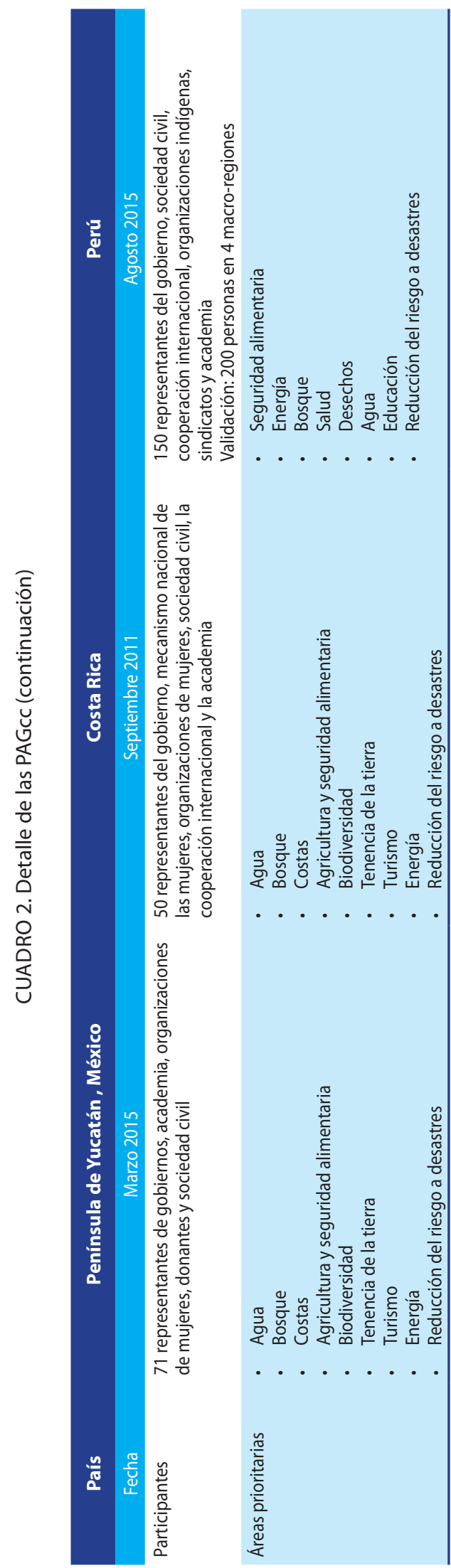

experiencia y conocimiento de la realidad en el terreno, así como ideas innovadoras para la acción. En la figura 2 detalla paso a paso el proceso de un PAGcc.

\section{Inventario de información}

Un PAGcc se fundamenta en las leyes y políticas nacionales existentes sobre cambio climático e igualdad de género. El proceso inicia con una solicitud formal de un gobierno ante la UICN; puede ser a petición del Ministerio de Medio Ambiente, o por el ente responsable de la política de cambio climático a nivel nacional. Seguidamente, se lleva a cabo una investigación para mapear el marco legislativo y programático, así como las iniciativas institucionales en áreas diversas como recursos naturales, aspectos socioeconómicos y sociodemográficos, industria, energía y finanzas entre otros. Este análisis abarca múltiples aspectos legales, incluido el derecho tradicional como el consuetudinario. Sobre la base de esta evaluación, se realizan entrevistas con los actores e instituciones claves y personas expertas, a fin de determinar las prioridades, desafíos y vacíos. La evaluación de las capacidades técnicas, los departamentos gubernamentales pertinentes, y otros grupos, completa este inventario de información y contribuye a contextualizar el documento.

\section{Nivelar el terreno}

Con el fin de mejorar la participación de las mujeres y las organizaciones de mujeres en el desarrollo de un PAGcc y en las actividades de cambio climático en general, se desarrolla una sesión de capacitación para fortalecer su conocimiento técnico en temas de cambio climático y para identificar las necesidades, prioridades e ideas de las mismas. Dependiendo del contexto del país, la representación en las sesiones de formación incluye mujeres con experiencia en política nacional y mundial, profesionales, mujeres líderes, campesinas, agricultoras, empresarias, entre otras.

\section{Captura de diversas voces y puntos de vista}

Como parte de un taller de múltiples actores, representantes de todos los sectores y departamentos de gobierno, instituciones no gubernamentales, academia, instituciones internacionales y grupos de la comunidad, entre otros, evalúan la situación actual del país en relación a género y cambio climático, prevén un escenario imaginario futuro en todos los sectores prioritarios, y acuerdan acciones futuras hacia ese escenario. Las 


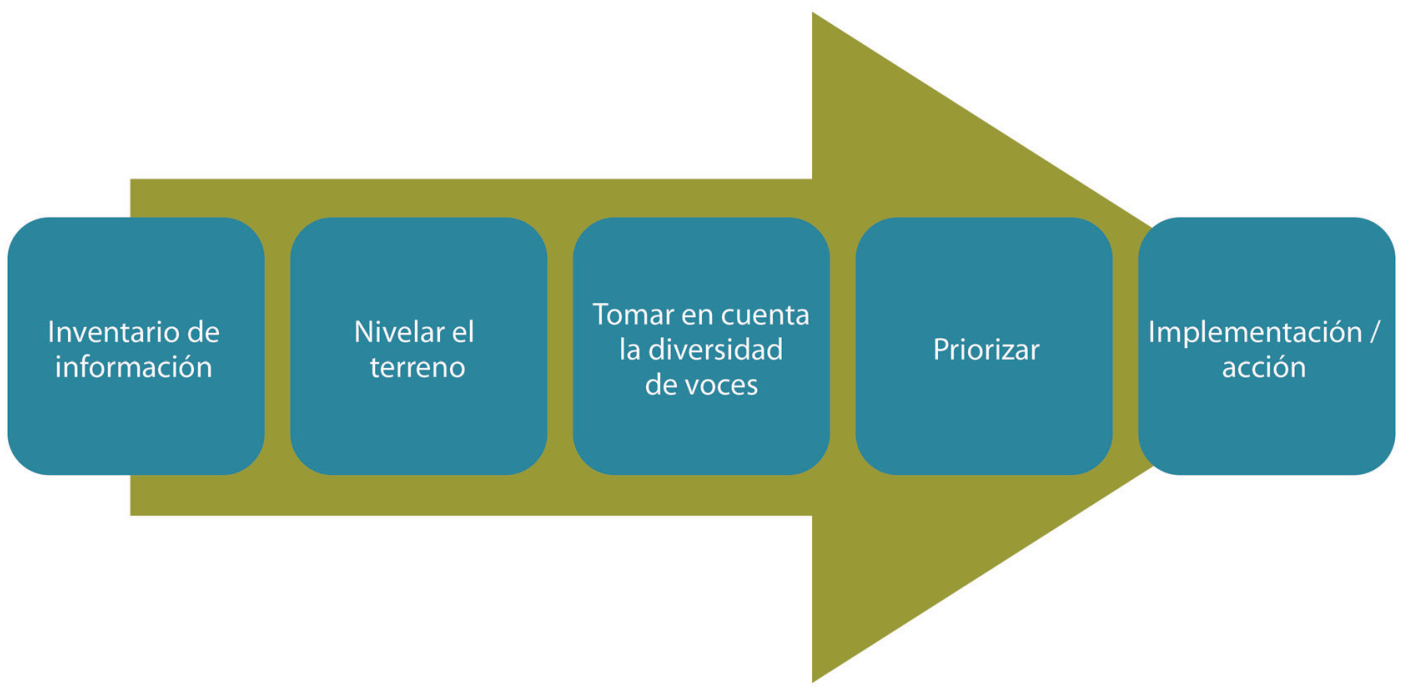

Fig. 2. Línea de Ruta PAGcc.

acciones caen dentro del ámbito de la adaptación y la mitigación en consonancia la planificación nacional para enfrentar el cambio climático.

\section{Priorizar}

Se conforma un equipo técnico, con representantes de los distintos sectores cuya tarea es convertir las acciones identificadas durante el taller en un plan de acción. Para ello se retoma el inventario de información elaborado y se priorizan las acciones para formular el borrador del PAGcc.

\section{Implementación}

El PAGcc se somete a un proceso de validación y consulta nacional por parte de las dependencias gubernamentales involucradas, con el objetivo de finalizar e internalizar el plan y trazar el curso para poner las acciones en marcha.

\section{Principios que fundamentan los PAGcc}

El principio subyacente de los PAGcc es la naturaleza transformadora que pueden tener las intervenciones que aseguran la igualdad de género en el contexto de cambio climático. Para lograr esto, el proceso se basa en seis principios, que la UICN reconoce como necesarios:

- INCLUIR a todos los grupos, sin distinción de edad, casta, etnia, religión o clase de manera que garantice su participación plena.
- INNOVAR en su propósito y proceso para llegar más allá de las soluciones comunes/tradicionales (por lo general circunscritas al hogar) y la búsqueda de propuestas e iniciativas nuevas e inspiradoras, mientras amplía las capacidades para un enfoque más global y comprensivo sobre el cambio climático.

- IMPULSAR mejoras en la calidad de vida de mujeres y hombres respecto a sus necesidades básicas (por ejemplo, agua), como a las necesidades estratégicas (por ejemplo, la tenencia de la tierra o la participación política). También reconoce las prioridades diferenciadas por sexo, los roles de género y el conocimiento necesario para enfrentar el cambio climático.

- IMPACTAR en la reducción de las emisiones antropogénicas.

- INTRODUCIR soluciones basadas en la naturaleza que no exceden los recursos naturales locales y nacionales y que contribuyan a amplificar los resultados del desarrollo sostenible.

- INCITAR a un cambio transformacional, promoviendo la igualdad de oportunidades entre mujeres y hombres para participar en soluciones, así como los medios necesarios para desarrollar las capacidades necesarias para promover un cambio real.

- INSPIRAR a los actores en todos los niveles a proponer hacer más allá del "mantenimiento del statu quo", demostrando que la aplicación de los compromisos por la igualdad de género y el cambio climático son posibles.

Como resultado, al aplicar estos principio, los PAGcc se construyen de manera más coherente y justa, lo cual 
les da características únicas, porque proponen una respuesta al desarrollo sostenible y el cambio climático mediante la participación no sólo de las mujeres, sino de comunidades enteras, sectores y gobiernos.

De esta forma, las acciones que se incluyen en los PAGcc: responden a una demanda sentida; hechas a la medida; diseñadas de abajo hacia arriba; funcionan como un vehículo para el desarrollo de capacidades y la coordinación dentro y entre las instituciones gubernamentales; son una plataforma para una mayor cooperación entre el gobierno y los diferentes sectores; incluyen una amplia gama de aspectos, según lo acordado por las partes interesadas; y contienen acciones e indicadores a múltiples niveles, desde el nivel del hogar hasta la política nacional tanto a nivel rural como urbano.
Pero las estrategias también son diversas en términos del contexto geo- político (área local, país, región); ecosistemas (tierras áridas / desiertos, zonas costeras, montañosa, etc.); abordan tanto adaptación como mitigación, o la vinculación de ambos y proponen soluciones basadas en el contexto del país.

Al mapear cada sector prioritario, acordar objetivos claves, identificar de acciones específicas y metas asociadas, los PAGcc presenta un plan de acción amplio sobre la igualdad de género y el cambio climático. El Cuadro 3 presenta ejemplos de una serie de países que han elaborado sus PAGcC.

Hasta la fecha, las discusiones de género y cambio climático tienden a concentrarse mayoritariamente en la vulnerabilidad de las mujeres y en la adaptación. Es

\section{CUADRO 3}

PAGcc Áreas prioritarias con ejemplos de acciones a seguir e indicadores

\begin{tabular}{ccl} 
País & \multicolumn{1}{c}{ Objetivos } & \multicolumn{1}{c}{$\begin{array}{c}\text { Agricultura y seguridad alimentaria } \\
\text { Acciones a seguir }\end{array}$} \\
Nepal & $\begin{array}{l}\text { Aumentar el acceso de } \\
\text { las mujeres a recursos } \\
\text { productivos (tierra, } \\
\text { semillas, fertilizantes, } \\
\text { crédito y equipo) }\end{array}$ & $\begin{array}{l}\text { Desarrollar una campaña nacional para incentivar y } \\
\text { fomentar la titulación de latierras a nombre del esposo } \\
\text { y la esposa (propiedad mancomunada de la tierra). }\end{array}$ \\
& $\begin{array}{l}\text { Construir resiliencia comunitaria en seguridad } \\
\text { alimentaria a través de la creación de bancos locales } \\
\text { de semillas que pertenecen y son administrados por } \\
\text { mujeres. }\end{array}$
\end{tabular}

Revisar las estrategias existentes que permitan el flujo de crédito de bancos públicos / comerciales e instituciones financieras para apoyar y ampliar el acceso de las mujeres al crédito.

Institucionalizar disposiciones alternativas para dar cabida a las mujeres, los grupos de mujeres, y las cooperativas que son incapaces de proporcionar las garantías necesarias para acceder al crédito agrícola.

Modificar y controlar disposiciones sobre subvenciones existentes por parte del gobierno en forma permanente para que las mujeres se beneficien equitativamente de ellos.

Cuba Incluir en el currículo universitario aspectos de género, la agricultura y el cambio climático

Promover mujeres agricultoras como facilitadores de cursos teóricos y prácticos sobre género y cambio climático en la agricultura en el ámbito universitario.

Apoyar la creación de grupos de mujeres en el cultivo y cosecha de especies que aumentan los rendimientos Aumentar la capacidad productiva de las mujeres para mitigar y adaptarse al cambio climático en respuesta al cambio climático, así como el rescate de los conocimientos tradicionales.

Promover el liderazgo de las mujeres en los bancos de semillas y las reservas genéticas.

\section{Indicadores de éxito}

Incremento en el número de registro de la propiedad mancomunada.

Número de grupos de mujeres utilizando y beneficiándose de los bancos de semillas

Número de crédito otorgado a las mujeres agricultoras.

Tasa de rendimiento en el crédito extendido.

Número de nuevas opciones colaterales alternativas puestas a disposición de las mujeres, los grupos de mujeres y las cooperativas por parte de bancos distintos de la tierra / vivienda / salario.

Aumento en la producción de alimentos basados en el acceso a los recursos productivos.

Número de mujeres campesinas que imparten cursos en centros de formación profesional en el sector agrícola.

Número de mujeres implementando tecnologías innovadoras para mitigar y adaptarse al cambio climático.

Número de bancos de semillas y reservas genéticas liderado por mujeres.

Estudios sobre el rendimiento y la innovación promovidos por las mujeres. 
Liberia Establecer un sistema Consultar con las partes interesadas en la selección de de monitoreo o en las zonas costeras en manos de las mujeres

"las" monitoras costeras (mujeres denunciantes).

Desarrollar términos de referencia para orientar la

$\begin{array}{cl}\text { Tanzania } & \text { Desarrollar programas } \\ & \text { / proyectos género- } \\ & \text { responsivos que } \\ & \text { aborden la adaptación } \\ & \text { al cambio climático en } \\ & \text { las costas. }\end{array}$
las costas. participación de las mujeres en la vigilancia de las zonas costeras. El sistema se basará en el uso de teléfonos móviles para las mujeres.

Establecer alianzas con empresas de telefonía móvil para apoyar / patrocinar este programa.

Capacitar y empoderar a las mujeres en la vigilancia costera.

Monitoreo de zonas costeras y medidas puestas en marcha con la participación de las mujeres.

Implementar proyectos / programas que incluya la adaptación al cambio climático en las zonas costeras (restauración de los bosques costeros y arrecifes de coral, restauración de dunas de arena, muros de contención, cabos, y la regeneración de playas).

Promover y fomentar la participación de las mujeres en los proyectos de manejo integrado costero.

Capacitar e integrar a las mujeres para que participen en estudios de investigación (por ejemplo, monitoreo y métodos de recopilación de datos; recolección de especies de flora y fauna; análisis físico-químico, estudios sobre las poblaciones de aves, peces, algas marinas, mamíferos y estudios sobre las propiedades medicinales del coral).

Establecer una red de organizaciones de mujeres dedicadas a la protección, gestión y desarrollo de las zonas costeras y marinas

Apoyar y desarrollar la capacidad de las organizaciones de mujeres para que puedan estar a cargo del monitoreo costero, la erosión de coral, el nivel del mar y la marea, la detección de la luz, entre otros.
Número de mujeres capacitadas en la vigilancia costera.

Número de incidentes reportadas por mujeres monitores.

Información desagregada sobre las actividades antropogénicas e impactos en las zonas costeras disponibles.

Número de planes de acción géneroresponsivos.

Número de proyectos relacionados a género implementados.

Número de mujeres que participan en proyectos de adaptación al cambio climático.

Mujeres y hombres capacitados en el uso sostenible de los recursos costeros.

Las mujeres y los hombres reciben beneficios del proyecto (beneficios productivos, capacitación o crédito).

Las mujeres y los hombres participan activamente en la conservación de los recursos marino-costeros. importante tener en cuenta que los PAGcc promueve las ideas directas de las mujeres, su compromiso y liderazgo de manera innovadora y su rol activo en aspectos relacionados con la mitigación. A través de un amplio abanico de temas de mitigación, los PAGcc en todo el mundo han producido nuevas formas de responder a las preocupaciones de género y cambio climático. Ejemplos de estos esfuerzos se detallan en el Cuadro 4.

En todo el mundo los PAGcc están empezando a revelar el potencial transformador de la igualdad de género. En Mozambique, el PAGcc fue el catalizador que incidió en la inclusión de medidas de igualdad de género en el desarrollo del Programa Estratégico del país en la iniciativa de la adaptación al cambio climático para los
Fondos de Inversión Climática. En Jordania, el PAGcc inspiró al gobierno a declarar la igualdad de género como una prioridad nacional en respuesta al cambio climático, esto fue evidente en la tercera comunicación nacional del 2014 a la CMNUCC y en su INDC del 2015, en este último se menciona específicamente el proceso de la PAGcc y su influencia. Lo mismo sucede con los INDCs de Liberia y Perú.

La demanda de los PAGcc ha crecido en los últimos cinco años, sobre todo en respuesta a los impactos al cambio climático que viven los países en desarrollo, así como los mandatos para hacerle frente a las consideraciones de género en la política mundial y en la planificación nacional. 


\section{CUADRO 4}

Liderazgo de las mujeres en mitigación: Ejemplos de actividades innovadoras en los PAGcc

Sector

Transporte
País

Egipto

Bangladesh

Energía

(B)

Bosques

Tanzania

Manejo de desechos Perú

Nepal

Energía
Actividades de mitigación por las mujeres

Reducir las emisiones y ampliar los medios de vida mediante la creación de una red de taxis acuáticos administrado por las mujeres en el Nilo.

Venta de tiquetes y zonas de descanso seguras para las mujeres en las terminales de autobuses y trenes, y la introducción de pases de un día y un billete común para autobuses, trenes y barcos.

Mozambique Reducir las emisiones y la dependencia de las fuentes de combustibles tradicionales mediante el establecimiento y funcionamiento de tiendas de energía administradas por las mujeres de la comunidad.

Jordania Reducir la huella de carbono nacional mediante la realización de campañas dirigidas a las mujeres para reducir las emisiones en los hogares con la mejora de la eficiencia energética y los patrones de consumo

Abogar por la gobernanza forestal sostenible a través de campañas de educación para las mujeres y niñas en el sector forestal.

Identificar e implementar esquemas de distribución de beneficios para las mujeres bajo REDD+.

Creación del programa "Barrios Limpios" que establece una relación de colaboración entre los vecinos y los recicladores locales.

Desarrollar programas de manejo de residuos y salud a través de un programa no tradicional (es decir, el reciclaje, la reutilización) que brinde trabajo específicamente para las mujeres.

Bangladesh Crear e incluir una metodología para la reducción del consumo en el currículo escolar nacional.

Mozambique Establecer un fondo interministerial para las mujeres que les facilite el acceso a las tecnologías verdes

Haití Capacitar a las mujeres para que reparen los componentes de los sistemas fotovoltaicos y construyan cocinas mejoradas.

Transferencia de tecnología Haití
Desarrollar la capacidad de hombres y mujeres en el diseño de edificios verdes.

\section{Estrategias regionales}

La implementación del marco y las políticas de cambio climático internacional comienzan a tomar forma a escala regional, los PAGcc han sido tomados como ejemplo para convertirse en otra estrategia para facilitar estos procesos. Tres PAGccs (interestatales) regionales se han desarrollado a partir del 2015, incluyendo la Liga de Estados Árabes, América Central y la Península de Yucatán.

La región representada por la Liga de Estados Árabes ha participado activamente en las sesiones de orientación sobre género y clima impartidos por UICN/GGCA, convirtiéndose en firmes defensores de la igualdad de género en las negociaciones internacionales sobre el cambio climático. El desarrollo de la capacidad de los delegados de la Liga de los Estados Árabes, en especial, el apoyo a las delegadas, ha fortalecido el conocimiento e identificó la necesidad de que las políticas climáticas con perspectiva de género se elaboraran. Una fuerte relación entre la UICN y sus Estados miembros, apoyada por la Oficina Regional de la UICN para Asia Occidental, favoreció la incorporación de género y la toma de decisiones, e impulsó el desarrollo de un marco regional sobre cambio climático por expertos locales de género. El desarrollo del marco regional demuestra una dedicación por los tomadores de decisiones en la región para valorar el género y la visión de las mujeres como agentes de cambio que contribuyen a la capacidad de los países contra el cambio climático. Como resultado del PAGcc Regional de la Liga Árabe, el género está incluido como un tema permanente en el programa de preparación de material técnico para las reuniones del Consejo de Ministros Árabes responsables del medio ambiente (parte de la Liga de los Estados Árabes) (UICN, 2012).

En América Central, la vulnerabilidad regional y las capacidades y necesidades de las mujeres y los hombres que enfrentan el cambio climático provocaron que los líderes nacionales prestaran atención a las diferentes prioridades de las mujeres y los hombres. Además escucharon las voces de las mujeres, quienes reconocieron y catalogaron los diferentes impactos que han ido 
en aumento debido a los desastres vinculados con el cambio climático.

\section{Otra iniciativa impresionante!}

Expertos(as) de género en toda América Central comprometidos con las mujeres locales y rurales, con base en sus experiencias y recomendaciones, formaron un comité técnico para acercarse al Sistema de Integración Centroamericana (SICA), institución encargada de elaborar una estrategia regional de cambio climático, con una propuesta formal de estrategia de cambio climático que incluyó el enfoque de género. La UICN fue invitada al proceso y proporcionó el desarrollo de capacidades para mejorar el impacto de la comisión a nivel regional y mundial. El comité también examinó la estrategia de cambio climático y el proyecto identificando vacíos significativos en el abordaje de las preocupaciones de las mujeres. La estrategia fue confirmada en una reunión con la red de Mujeres Ministras, aprobada y legalizada por el Consejo de Ministros de la Comisión Centroamericana de Ambiente y Desarrollo. Lo anterior generó presión política para llevar a la incorporación el enfoque de género como un mandato en la Declaración de los jefes de Estado, en relación con la estrategia de cambio climático para la región en julio de 2010, que luego llevó el mensaje de la igualdad y equidad de género a la escena internacional para los resultados de las negociaciones sobre el clima de la CMNUCC a finales de ese año en Cancún.

Los procesos políticos regionales y mundiales son valiosos, pero es igualmente importante encontrar maneras de implementar la estrategia en lugares específicos y validar las acciones con las comunidades que comparten sus experiencias en un principio. El éxito de esta estrategia regional puede depender de la colaboración de los gobiernos de América Central con las ONG en coordinación con el movimiento de mujeres en la región.

En marzo de 2015, la UICN, con el apoyo de la del Ministerio Federal Alemán de Medio Ambiente, Conservación de la Naturaleza, Construcción y Seguridad Nuclear (BMUB), la Agencia Noruega de Cooperación para el Desarrollo (NORAD) y el Departamento para el Desarrollo Internacional (DFID) - colaboraron con los gobiernos de los estados de la Península de Yucatán con el objetivo de desarrollar directrices regionales para la integración de la perspectiva de género en los procesos de adaptación y mitigación para la Península de Yucatán. Esta es la primera región (intraestatal) de México que muestra el compromiso de cumplir los acuerdos y mandatos firmados por México ante la CMNUCC en relación con la inclusión de género en programas de cambio climático.

\section{Recomendaciones}

Las acciones nacionales y regionales demuestran pasos importantes en la dirección correcta para promover la igualdad de género dentro de la agenda del cambio climático. Hasta hace poco, a la luz de los relativamente nuevos compromisos, las políticas y planes nacionales rara vez reflejaban el carácter global de estos temas. La sociedad civil internacional ha centrado su esfuerzo en garantizar que los aspectos de igualdad de género y empoderamiento de las mujeres se integren en los procesos de decisión de cambio climático, y en la planificación intersectorial, donde gran parte en la inversión de la promoción de la igualdad de género se ha realizado. Hasta hoy día los avances han sido pocos, a pesar del apoyo de la CMNUCC, pero buenas oportunidades existen para trabajar estos aspectos en el futuro próximo.

A medida que los países avanzan en la implementación de una gama de estrategias, incluyendo el desarrollo de políticas, programas y proyectos, habrá un incremento de ejemplos de cómo el enfoque de género ha sido un catalizador para el éxito, ya que las consideraciones de género se integran cada vez más eficaz y coherentemente en la planificación del desarrollo resiliente y bajo en emisiones. Para garantizar que la política climática, la planificación y los indicadores sean género responsivos y que se continúe su desarrollo e implementación para lograr resultados óptimos las siguientes recomendaciones debe ser consideradas:

- Continuar el desarrollo de las capacidades de las partes interesadas, para entender los vínculos entre género y cambio climático, en todos los niveles. Se debe reconocer la necesidad de vincular el marco internacional sobre cambio climático con la articulación en el ámbito nacional, regional y local, la creación de capacidades es esencial en todos los sectores, para construir puentes entre los distintos sectores y reconocer específicamente los derechos de la mujer y los aspectos de igualdad de género.

- En paralelo: promover y destacar el valor de la igualdad de género y el empoderamiento de las mujeres en todos los programas, actividades de sectores especíicos e inversiones -tales como programas de inversión en agricultura- mediante la ejecución de actividades ya identificadas e incluidas en los PAGcc . Estas actividades y planes deben ser apoyadas por presupuestos y tecnologías género-responsivas. 
- Asegurar la inclusión de los mecanismos de la mujer (eje. Secretaria o Ministerios de la Mujer en la implementación, y en particular en la distribución de beneficios, - pero también en la inversión- a mujeres y organizaciones de mujeres para fortalecer su empoderamiento y permitirles el acceso más allá de las diversas barreras socio-culturales que enfrentan y las marginan de la participación en el desarrollo rural y urbano.

- Crear espacios y garantizar oportunidades para la participación de las mujeres en los procesos nacionales de toma de decisiones sobre cambio climático tanto en aspectos relacionados a la adaptación así como a la mitigación (ejem. energías renovables, REDD+)

- Movilizar, o aumentar, el acceso a los mecanismos de financiación para impulsar la aplicación de estrategias y planes de acción. En el pasado, los países han declarado que no existían fondos o mandatos para incorporar la perspectiva de género en el cambio climático, pero ahora existe la voluntad y la capacidad para aprovechar el financiamiento con enfoque de género a nivel nacional e internacional. Cabe destacar que en el presente documento todos los fondos relacionados con el cambio climático a nivel internacional cuentan con políticas y mandatos de género (Fondo para el Medio Ambiente Fondo Verde para el Clima, entre otros). El desafío consiste en desarrollar "los saberes- prácticos y metodológicos" en los diferentes implementadores para lograr resultados sociales positivos, especialmente para las mujeres y grupos de mujeres.

- Revisión de las políticas y estrategias nacionales de género y cambio climático, incluyendo los PAGcc ya desarrollados, y la actualización a la luz de las comunicaciones, mecanismos de financiación, particularmente el Fondo Verde para el Clima, que comienza al desembolsar los fondos en el marco de la CMNUCC.

- Involucrar a una amplia gama de socios que trabajan en materia de igualdad de género y el empoderamiento de las mujeres a entender mejor las causas fundamentales de la desigualdad de género, extraer lecciones de sus experiencias y consolidar alianzas entre los profesionales nacionales e internacionales para la adaptación al cambio climático y la mitigación. Basado en iniciativas anteriores y las que están en curso se puede aprender de los roles de las mujeres y los hombres en la mitigación del cambio climático y la adaptación. Aumentar el conocimiento e intercambio de lecciones y mejores prácticas a través de redes de aprendizaje, como el mecanismo de conocimiento para la adaptación.

- Capacitar y sensibilizar a las partes interesadas y el personal del proyecto en materia de género de la importancia de la igualdad de género y el empoderamiento de las mujeres no sólo por resultados equitativos eficientes y sostenibles, sino también para proteger y promover los derechos humanos. Los estudios de diagnóstico, como las estrategias de igualdad de género, realizados por expertos independientes de género, en consulta con los principales interesados, ayudan en la identificación de las brechas de género y en el cómo incluirse los aspectos de género en la planificación, ejecución y seguimiento de proyectos sobre cambio climático.

\section{REFERENCIAS}

Aguilar, L. (2009). Training manual on gender and climate change. San José, Costa Rica: IUCN, UNDP y GGCA.

Blomstrom, E., \& Burns, B. (2015). Global policy landscape: A supporting framework for gender-responsive action on climate change. En Aguilar, L., Granat, M. y Owren, C. (Eds.). Roots for the future: The landscape and way forward on gender and climate change. (p.p.47-80). Washington, D.C., EEUU: IUCN y GGCA.

CMNUCC. (2017). Achieving the goal of gender balance- Technical paper by the secretariat. FCCC/TP/2017/8. Bonn, Germany.

IUCN. (2012). The art of implementation: Gender strategies transforming national and regional climate change decision making. San José, Costa Rica: IUCN y GGCA.

UNFCCC-Subsidiary Body for Implementation. (2015). Report on the in-session workshop on gender-responsive climate policy with a focus on mitigation action and technology development and transfer. Recuperado de http://unfccc. int/resource/docs/2015/sbi/eng/12.pdf

UNFCCC. (2016). Acuerdo de Paris. Recuperado de http://unfccc. int/files/meetings/paris_nov_2015/application/pdf/paris_agreement_spanish_.pdf 